of mefloquine the cure rate was a remarkable $100 \%$. Our results indicate that at least 12 hours should elapse between the last dose of quinine and the dose of mefloquine to minimise gastrointestinal side effects. All the patients except two received $1.5 \mathrm{~g}$ mefloquine. The other two patients responded well although they received only $1.0 \mathrm{~g}$. Possibly the quininemefloquine regimen will prove to be just as effective and less toxic using the lower dose of mefloquine. The fact that no repeat attacks of falciparum malaria occurred during follow up in the patients who received quinine-mefloquine suggests that repeat attacks after the other regimens were recrudescences and not reinfections.

Sequential treatment with quinine and Fansidar is the most effective regimen using drugs that are, at the moment, commercially available. In this and a previous study, ${ }^{4}$ quinineFansidar had a $96 \%(342 / 357)$ cure rate. With both quininemefloquine and quinine-Fansidar at least four doses of quinine were needed to produce a consistently rapid clinical improvement. To achieve a virtually $100 \%$ cure rate with quinineFansidar at least 10 doses of quinine would be required.

The method developed in our studies of using mefloquine or Fansidar after a course of quinine receives support from studies by Peters et al of malaria in rodents. ${ }^{11}$ They concluded that mefloquine may with time lose its effectiveness against chloroquine-resistant malaria parasites and that by using it in combination with other antimalarial drugs its useful life could be extended. Sequential quinine-mefloquine is the most effective treatment yet tested for chloroquine-resistant falciparum malaria of all degrees of severity, and mefloquine alone will prove valuable for the single-dose treatment of mild falciparum malaria. ${ }^{\circ}$

\section{References}

${ }^{1}$ Harinasuta, T, Suntharasamai, P, and Viravan, C, Lancet, 1965, 2, 657. 2 Colwell, E J, et al, American fournal of Tropical Medicine and Hygiene, $1972,21,6$.

${ }^{3}$ Hall, A P, et al, American Fournal of Tropical Medicine and Hygienes 1975, 24, 575.

4 Hall, A P, et al, British Medical fournal, 1975, 2, 15.

Trenholme, G M, et al, Science, 1975, 190, 792.

- Hall, A P, et al. Submitted for publication.

' Doberstyn, E B, et al, American fournal of Tropical Medicine and Hygiene, 1976, 25, 14.

${ }^{8}$ Hall, A P, et al, Transactions of the Royal Society of Tropical Medicine and Hygiene, 1975, 69, 342.

- Hall, A P, et al, British Medical fournal, 1975, 2, 12.

10 World Health Organisation. Chemotherapy of Malaria, Technical Reports Series No 375, p 42. Geneva, World Health Organisation, 1967.

11 Peters, W, Portus, J H, and Robinson, B L, Annals of Tropical Medicine and Parasitology, 1975, 69, 155.

(Accepted 1 Apri! 1977)

\title{
Severe systemic and pyogenic infections with Bacillus cereus
}

\author{
P C B TURNBULL, T A FRENCH, ELIZABETH G DOWSETT
}

British Medical fournal, 1977, 1, 1628-1629

Bacillus cereus is becoming well known as an aetiological agent of food poisoning but it has also caused infections not related to food-for example, bronchopneumonia, ${ }^{1-3}$ bacteraemia and septicaemia, ${ }^{4-6}$ and meningitis. ${ }^{5} 7$ The following two case reports add to the increasing awareness that $B$ cereus should not be regarded as an inconsequential contaminant. Toxigenicity tests on $B$ cereus isolates from these cases yielded important evidence on the mechanism of $B$ cereus pathogenesis.

\section{Case reports}

CASE 1

A 1320-g girl infant was grunting on breech delivery at 32 weeks' gestation and was later thought to have the respiratory distress

Food Hygiene Laboratory, Central Public Health Laboratory, London NW9 5HT

P C B TURNBULL, MS, PHD, senior scientist

Children's Hospital, Ladywood, Birmingham B16 8ET

T A FRENCH, MB, MRCPATH, senior registrar (now consultant pathologist, Central Pathology Laboratory, Hartshill, Stoke-on-Trent)

Basildon Hospital, Basildon, Essex

ELIZABETH G DOWSETT, MB, DIPBACT, consultant microbiologist syndrome. An umbilical catheter was inserted the day after delivery. The abdomen became progressively distended and the meconium blood-stained. Apnoeic attacks began. Abdominal radiography showed gas under the diaphragm. At laparotomy free bile-stained fluid was found in the peritoneal cavity and a 7-mm perforation was seen on the antimesenteric border of the ileum about $7 \mathrm{~cm}$ from the ileocaecal valve. The mesentery at this point was torn almost to its root. Loop ileostomy was performed. Apnoeic attacks continued, and the second day after delivery she became jaundiced. Next day a central venous catheter was inserted. On the fourth day she had apnoeic attacks and convulsions affecting both sides, and despite intravenous gentamicin and ampicillin and general supportive care she died. At necropsy the mesenteric defect was confirmed at the site of the repaired perforation. A further mesenteric defect at the duodenojejunal flexure $1.2 \mathrm{~cm}$ in diameter was also found. There was no appreciable peritonitis. The brain was unusually soft and was examined after fixation. The softening was widespread and was particularly noticeable in the cerebral cortex and basal ganglia, where it was red-black, resembling the colour of altered blood. There was blood in the lateral ventricle. No other abnormalities were detected.

Histologically the lungs showed acute inflammatory foci including Gram-positive bacilli and Gram-positive cocci related to fibrinoid necrosis of arteries. In the brain there were extensive areas of necrosis with an eosinophilic appearance containing numerous Gram-positive bacilli but no inflammatory infiltration. In the respiratory tract in the region of the subglottis there was necrotising ulceration with numerous Gram-positive bacilli.

Blood taken on the fourth day before death yielded 2400 colonies of faecal streptococci and 20 colonies of Gram-positive bacilli per $\mathrm{ml}$. Large numbers of Gram-positive bacilli together with small numbers of Escherichia coli and faecal streptococci and moderate numbers of Candida were isolated from the lungs. Moderate numbers of Grampositive bacilli and faecal streptococci and small numbers of $E$ coli were isolated from brain swabs. The Gram-positive bacilli were identified by the Food Hygiene Laboratory as $B$ cereus. 
CASE 2

A previously fit 17-year-old apprentice sustained a closed fracturedislocation of the right ankle in a motor-cycle accident. Open reduction was performed, a Rush nail inserted, and an above-knee plaster cast applied. During the next 48 hours his temperature rose to $38.5 \mathrm{C}$, the leg remained painful, and a haematoma developed at the site of the wound. Re-exploration of the operation site on the fourth day disclosed purulent, foul-smelling discharge. Multiple blisters were noted over the right medial malleolus and calf. There was also an uninfected haemarthrosis of the right knee, which was later aspirated. Flucloxacillin was given without benefit (culture of a specimen taken from the operation site at this time later grew Proteus mirabilis).

Six days after injury the patient remained feverish and haematuria was noted in the absence of injury to the urinary tract. He was pale and restless and had gross oedema and discoloration of the right leg extending up to the thigh. A copious thin discharge with a musty smell was seeping through the plaster. A window was cut over the wound and the sutures were removed. Direct Gram-staining of material from the wound disclosed numerous Gram-positive sporing bacilli and scanty Gram-negative bacilli. He was forthwith treated as a case of gas gangrene. The haemoglobin had fallen $5 \mathrm{~g} / \mathrm{dl}$ since admission and a blood transfusion was ordered, and he was given $10 \mathrm{MU}$ penicillin daily (plus $240 \mathrm{mg}$ gentamicin for the proteus infection). The plaster was removed and the wound treated with hydrogen peroxide soaks. Within 48 hours he became afebrile and the haematuria ceased, and within one week he was well enough to have the wound resutured and the leg re-encased in plaster. He was discharged on the 26th day and his subsequent course was uneventful.

Culture samples taken on the sixth day and from the deep recesses of the wound on the 17 th day grew $\mathrm{Pr}$, morgani and $B$ cereus. The $B$ cereus was sensitive to erythromycin, gentamicin, and fusidic acid but resistant to penicillin, sulphonamides, and trimethoprim. Clostridia and other anaerobes were not isolated despite extended incubation and selective techniques. A few colonies of Streptococcus faecalis were also isolated on the sixth day. The results of blood cultures taken on the sixth day were negative.

\section{Bacteriological and toxicological findings}

The $B$ cereus isolate $(\mathrm{F} 2141 / 74)$ in case 1 was particularly noted during a study ${ }^{8}$ on enterotoxin production by strains that, apart from the strain from this infant, were derived from foods or food-poisoning cases. Concentrated cell-free culture filtrates of this strain caused appreciable mucosal necrosis within a few hours in ligated rabbitileum ("rabbit-loop") tests. The toxin responsible for this may also have caused the cerebral necrosis and necrotic ulceration of the respiratory tract.

In a follow-up study ${ }^{9}$ on $11 \mathrm{~B}$ cereus strains isolated from various clinical conditions it was found that the strains could be placed on a $0-4+$ scale according to the extent of necrotic damage they could produce in rabbit ileum or guinea-pig skin. Cell-free filtrates of strain F2141/74, prepared as described, ${ }^{8}{ }^{9}$ were found consistently to produce a $2+$ level of necrosis in both test systems.

Based on colonial morphology two isolates (F4581A/76, F4581B/76) of $B$ cereus were obtained from the culture in case 2 . These were indistinguishable serologically, biochemically, and, apart from a slight difference in tetracycline resistance, in their antibiotic susceptibility pattern (Oxoid Multodisc No 11-14C). In both the rabbit-loop and guinea-pig skin tests F4581A was consistently innocuous, while F4581B produced up to $3+$ mucosal necrosis in rabbit ileum and $4+$ necrosis in guinea-pig skin. We therefore propose that F4581B was responsible for the gangrenous nature of the infection in case 2 . Swabs from the environments of the operating theatre and plaster room where the patient was treated yielded three isolates of $B$ cereus but none was identical with the strain obtained from the patient.

\section{Discussion}

As a group, $B$ cereus organisms have long been known to produce a haemolysin immunologically related to streptolysin $\mathrm{O}$, phospholipases, proteolytic enzymes, penicillinase, lecithinase, and a lethal toxin. ${ }^{10}$ Members of the species are now recognised as agents of distinct vômiting and diarrhoeal food-poisoning syndromes, and evidence has been provided that distinct enterotoxins are responsible for the two syndromes. ${ }^{11}$ The diarrhoeal toxin has been partially characterised and shown to be distinct from the haemolysin and lecithinase, ${ }^{12}$ but it is thought that the necrotic, lethal, and diarrhoeal activities may be due to a single toxin.

With regard to the isolation of streptococci in case 1 , there have been several reports of necrotising local streptococcal infection or "streptococcal gangrene,"13 so that possibly the streptococci had a role in the pathological picture described. There is similar doubt about the role of proteus in case 2 . Proteus species, although often found in mixed culture in wounds and burns, can be pyogenic agents in their own right, and it was noted that the appearance of the brain in case 1 was similar to that in nine cases of proteus encephalitis at the same hospital. ${ }^{14}$ On the other hand, proteus infection is unlikely to explain the skin discoloration, gross oedema, and haemolytic manifestations of the infection in case 2 .

In neither case was the source of the $B$ cereus established. In the first case suggested portals of entry were the umbilical catheter, perforated ileum, and necrotic subglottis; ischaemic necrosis associated with hypoxia may have been a predisposing factor in localising the infection. ${ }^{15}$ In the second case it was suggested that the incriminated organisms were derived from the patient's skin and clothing and that the spores had survived attempts at skin disinfection. Spores of Bacillus species are highly resistant to commonly used skin disinfectants: in preparing skin for surgery by careful cleansing with detergent followed by the application of alcoholic iodine or iodophor, the importance of the cleansing step to remove spores cannot be overemphasised. Bacillus species have been isolated several times from blood cultures, necropsy cultures, and burns and occasionally, as in case 2 , from orthopaedic wounds in hospitals in the Essex area but have previously been considered to be contaminants.

In contrast to the closely related pathogen $B$ anthracis, for which penicillin is the drug of choice, $B$ cereus organisms are $\beta$-lactamase producers, and the penicillin administered in case 2 is unlikely to have played an important part in the patient's recovery. Gentamicin therefore appears to be the best choice, but in the presence of concurrent streptococcal infection, as in case 1 , additional antibiotic cover with an agent other than a penicillin would be necessary.

We thank Mr N Greville, consultant orthopaedic surgeon, South Essex District Hospitals, for the report on case 2, and Jennifer $M$ Parry, Food Hygiene Laboratory, for technical help.

\section{References}

${ }^{1}$ Coonrod, J D, et al, American Review of Respiratory Diseases, 1971, 103, 711.

${ }^{2}$ Feldman, S, and Pearson, T, Clinical Pediatrics, 1974, 13, 649.

3 Leff, A, et al, American Review of Respiratory Diseases, 1977, 115, 151.

4 Curtis, J R, Lancet, 1967, 1, 136.

5 Leffert, H L, Baptist, J N, and Gidez, L I, Fournal of Infectious Diseases, $1970,122,547$.

6 Goullet, P, and Pepin, H, Lancet, 1974, 1, 761.

${ }^{7}$ Raphael, S S, and Donaghue, M, Canadian Medical Association fournal, 1976, 115, 207.

8 Turnbull, P C B, fournal of Clinical Pathology, 1976, 29, 941.

- Turnbull, P C B, et al, British fournal of Experimental Pathology, 1977, 58, 273.

10 Bonventre, P F, and Johnson, C E, in Microbial Toxins, ed S J Ajl et al, vol 3, 415. New York, Academic Press, 1970.

${ }_{11}$ Melling, J, et al, fournal of Clinical Pathology, 1976, 29, 938.

12 Spira, W M, and Goepfert, J M, Canadian fournal of Microbiology, 1975, 21, 1236.

13 Wilson, G S, and Miles, A, Topley and Wilson's Principles of Bacteriology, Virology and Immunity, 6th edn, vol 2, p 1913. London, Edward Arnold, 1975.

14 Shortland-Webb, W R, fournal of Clinical Pathology, 1968, 21, 422.

15 Armstrong, D, and Norman, M G, Archives of Disease in Childhood, 1974, 49, 367.

(Accepted 14 April 1977) 